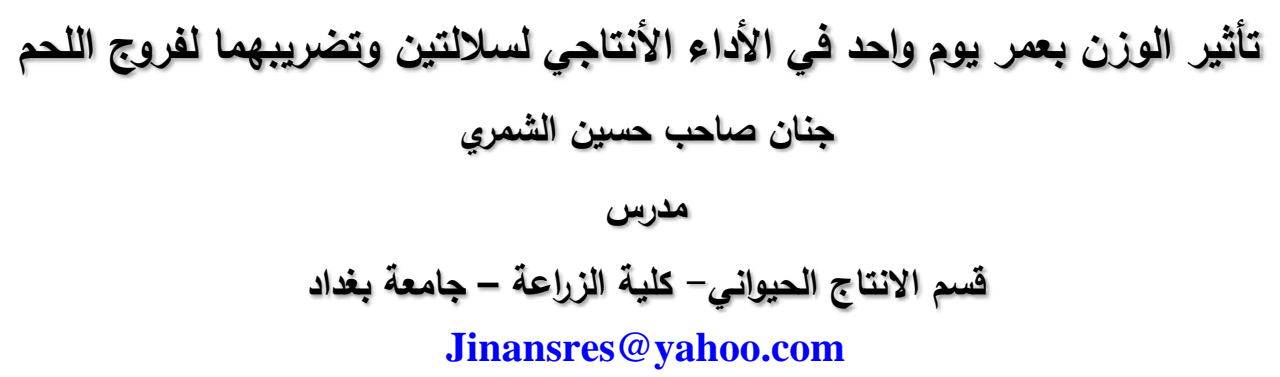

المستخلص

اجريث الدراسة في حقل الطيور الداجنة التابع لقسم الانتاج الحيواني /كلية الزراعة /جامعة بغداد للفترة من 1-4-2014 لغاية 12-5-

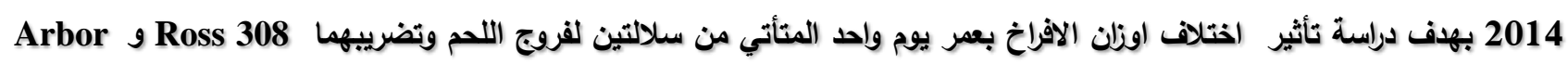

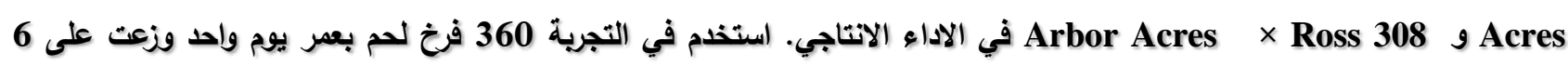

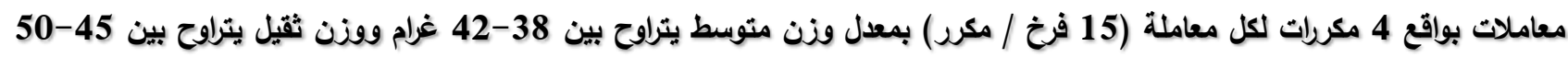

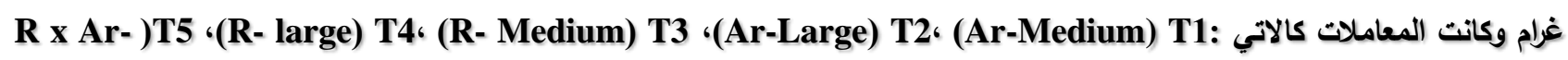

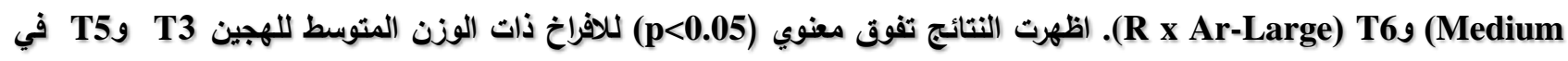

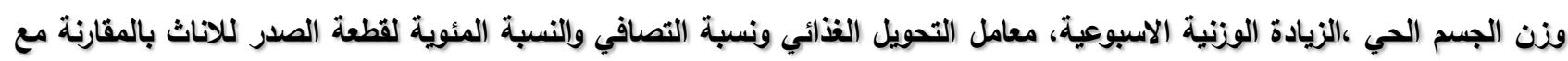
الافراخ ذات الاوزان الثقيلة. فيما لم يلاحظ فارق معنوي بين السلالات ولابين الجنسين بالنسبة لقطعة الصدر والقطعيات الثانوية للأبائح. نستنتج من ذلك ان الافراخ ذات الاوزلن المتوسطة اعطت وزن حي اعلى او مساوي للافراخ ذات الاوزلن الثقيلة فيما حسن التضريب بين الهجن الاداء الانتاجي لفروج اللحم. الكلمات المفتاحية: السلالات، وزن الفرخ ،الاداء الانتاجي.

The Iraqi Journal of Agricultural Sciences - 47(5):1290-1297, 2016 AL-Shamire

EFFECT OF DAY OLD CHICKS WEIGHT ON PRODUCTIVE PERFORMANCE OF TWO BROILER STRAINS AND THEIR CROSSING

Jinan S. H. AL-Shamire

Lecturer

Jinansres@yahoo.com

Dept. of Animal Production- College of Agriculture/University of Baghdad

\title{
ABSTRACT
}

This experment was conducted at the Poultry Farm‘Animal production Dept./College of Agriculture. University of Baghdad from 1-4-2014 to 12-5-2014. to investigate the potential effect of day old chicks weight produced from two broiler strains and their crossing Ross 308, Arbor Acres and Ross $308 \mathrm{x}$ Arbor Acres on productive performance . A total of 360 day all chicks were distributed randomly to 6 groups with 4 replicate pens )15 birds / pens). The experimental groups were: $T 1=$ Ar-medium; $T 2=$ Ar - Large ; T3= R-medium; T4= R -Large; T5= R - Arb-medium; T6= R - Ar-Large. Results showed that body weight, weekly average gain, feed conversion ratio, dressing percentage and relative breast weight in female were significantly $(p<0.05)$ better in group T3 and T5 compared to heavy chicks . While there was no significant differences between sex or strain for breast relative weight and other cuts. It could be concluded from this study that chicks with medium weight gave better or equal live weight compared to heaver strain however crossing between hybrids improved productive performance of broilers .

-Key wards: strain , chick weight, productive performance. 
دراسة على ثلاث فئات وزنية من الافراخ (صغيرة ، متوسطة المقدمة

وكبيرة). لاحظ Abiola وآخرون (4) ان الافراخ في المرحلة الاولى من (0- 4 اسبوع) حافظت على فئاتها الحجمية اما في المرحلة الثانية من (5-8 اسبوع) حصل ثقوقاً معنوياً في معدل وزن الجسم الحي للافراخ متوسطة الحجم واعطت اوزان مشابهة للافراخ الكبيرة. في حين لم تجد Alzawbaii (3) تأثير معنوي لوزن الفرخ على الوزن عند التسويق لمجموعتي الافراخ المتوسطة والكبيرة الحجم. لذلك جاءت هذه الدراسة لتقييم الاداء الانتاجي لسلالات فروج اللحم سلالة Ross 308 بين Arbor Acres × Ross 308 ولافراخ مختلفة الاوزان ناتجة من بيض تفقيس متوسط وكبير الحجم.

\section{المواد وطرق}

اجريت الدراسة في حقل الطيور الداجنة التابع لكلية الزراعة في ابو غربب وذلك للفترة من 1-4-2014 لغاية 12-52014 حيث استخدم في البحث 360 فرخ فروج لحم بعمر يوم واحد ناتجة من بيض تفقيس كبير الحجم يثراوح بين (68) - 71 غم) ومتوسط الحجم يتراوح بين (64.8 - 65.3 غم) تم الحصول عليه من الهيئة العامة للبحوث الزراعية - ابو غربب من امهات فروج لحم بعمر 52 اسبوع لسلالثين هما Ross 308 و Arbor Acres والتضريب بينهما وكانت الافراخ الناتجة بوزن متوسط (38-42 غرام) وثقيل (4550 غرام). شملت التجربة 6 معاملات بواقع 4 مكررات لكل معاملة (15 فرخ/ مكرر) وزعت المعاملات كالاتي:T1 T2 الفراخ منوسطة الوزن (Ar-M) Arbor Acres T3 الفراخ ثقيلة الوزن Ar-L) Arbor Acres (Aر) سلالة R-M) Ross 308 افراخ منوسطة الوزن T4 سلالة Arbor افراخ ثقيلة الوزن T5-L)Ross 308 سلالة (R) (R-Ar-M) Acres× Ross 308 (R-Ar-L) Arbor Acres× Ross 308 سلالة T6 افراخ ثقيلة الوزن. غذيت الافراخ على عليقة بادئ موحدة من (1-21 يوم) ثم استبدلت بعليقة نمو بعمر (22-42 يوم)
تتحصر اهمية صناعة الطيور الداجنة في الوقت الحاضر بتوفير منتجاتها الاساسية كاللحم والبيض وتعد نوعية الافراخ الجيدة ضرورية للمربي حيث تشكل اهمية كبيرة في دخله لكون المربي يرغب في الحصول على افراخ تتميز بكفاءة نمو عالية وحيوية عالية وانتاج عالي من لحم الصدر (17) و16). وقد عملت الثركات المتخصصة لتحسين الطيور الداجنة من الناحية الوراثية بأستخدام طرائق تمكن من استثمار التباين الوراثي غير التجمعي المسؤول عن قوة الهجين في التضريبات بين المجاميع الوراثية المختلفة (9) للوصول الى افضل التوليفات بين الخطوط او السلالات وتحديد الميزات الرئيسية لكل خط ومن ثم تحسين الاداء الانتاجي للهجن الناتجة ففي دراسة لل Al-Khailany (2) لمقارنة الاداء الانتاجي للنسل الناتج من سلالات لوهمان وفابرو ويوربرد التي جرى عليها قلش اجباري حيث تفوقت سلالة لوهمان على باقي السلالات في وزن الجسم الحي عند عمر 49 يوم واعطت 2242.3 و 1950 و 1807.5 غرام عesseraud على التوالي. ودراسة اخرى قارن فيها QL وآخرون(15) بين خطين من هجن فروج اللحم التجاري" (خط منتخب لعضلة الصدر وانخفاض دهن البطن) و LC خط عشوائي لم يمارس فيه الانتخاب ،حيث تفوقت طيور ، في وزن الجسم الحي ومعدل الزيادة الوزنية Q الخط لفية وفي دراسة ايضاً ل Ahmed (1) لتقييم النسل الناتج من سلالتي Hubbard و 20ss 308 وجد فروق معنوية في وزن الجسم الحي والزيادة الوزنية لصالح 308 Ross. ومن ولن المعروف بأن وزن بيض التفقيس يؤثز على النمو الجنيني وامكانية النمو بعد الفقس حيث تتاولت دراسات عديدة نأثير وزن الفرخ في معدل وزن الجسم الحي منها اظهرت علاقة موجبة عند الاعمار المختلفة فيما اشارت دراسات اخرى الى ان معدل وزن الافراخ الناتجة من بيض متوسط الحجم كانت تعطي افضل وزن حي (5). ولاحظ Laughlin (10) وجود علاقة قوية بين وزن الفرخ ووزن الجسم عند التسويق، وفي 


\section{النتائج والمناقشة}

تشير نتائج الجدول 2 الى تفوق معنوي للمعاملتين T2 وT5 في معدل وزن الجسم الحي خلال الاسبوع الاول على باقي المعاملات. في حين تفوقت المعاملة T5 على معاملات التجربة في الاسبوع الثاني وسجلت المعاملة T3 اقل معدل وزن جسم حي وكان 405.27 غم. فيما عادت المعاملة T2 للتفوق معنوياً ( على باقي المعاملات خلال الاسبوع الثالث. في حين تفوقت المعاملة T3 على معاملات التجربة في الاسبوع الرابع وسجلت معدل وزن جسم حي 1535.83 غم اما في Ross ) T3 الاسبوع الخامس والسادس فقد تفوقت المعاملتين 308 منوسط الوزن) و Arbor Acres x Ross 308) T5 متوسط الوزن) معنوياً (P<0.05) على معاملات التجربة ولم تختلف المعاملة T6 عنهما معنوياً خلال الاسبوع السادس من التجربة. وقد يعزى تفوق المعاملتين T3 و T5 وكلاهما افراخ منوسطة الوزن خلال الاسابيع 4 - 6 الى الوزن الفيزيائي للفرخ والذي يمثل وزن الجسم الحقيقي مضافاً له وزن الصفار المتبقي والذي يتراوح بين 2- 8 غرام فقد يكون وزن الفرخ الكبير يعود اللى وزن كيس الصفار الكبير فيما قد تكون الافراخ ذات الاوزان المتوسطة ذات كيس صفار قليل الوزن وهذا مالاحظه Abiola وآخرون (4) بأن الافراخ حافظت على اوزانها خلال المرحلة الاولى من (0 - 4) فيما تفوقت ذات الفئة المتوسطة خلال المرحلة النهائية من 5- 8 اسبوع ولم تختلف معنوياً عن الفئة ثقيلة الوزن وهذه النتيجة تطابقت مع AL-zawbaii (1) حيث لم تناحظ فارق معنوي في الوزن الحي بين الافراخ المتوسطة والثقيلة الوزن. وقد يعود تفوق الافراخ ذات الاوزان المتوسطة الى وزن بيض التفقيس المتوسط الحجم والذي يفضل استخدامه في عملية التفقيس التي يستبعد فيها البيض الكبير والذي تكون فيه نسبة البياض الى الصفار اكثز من 2:1 او قد يكون سمك القشرة اقل لانها تتوزع على مساحة اكبر وهذه تؤثر على حيوية الجنين وعلى كمية الكالسيوم التي يحصل عليها اثثاء الفقس
الجدول1. زودت الافراخ بالعلف والماء بصورة حرة وبأستخدام معالف يدوية معلقة ومناهل اوتماتيكية معلقة. وزنت الافراخ اسبوعياً وحسب العلف المستهلك،الزيادة الوزنية ومعامل التحويل الغذائي اسبوعياً.في نهاية التجربة ذبح ذكر وانثى من كل مكرر وحسب وزن الذبيحة ونسبة التصافي بدون الاعضاء المأكولة والقطعيات الرئيسية (الصدر والافخاذ) والثانوية (الظهر والاجنحة والرقبة). جدول 1. نسب المواد العلقية الداخلة في تكوين علائق البادىء والنمو لفروج اللحم والتركيب الكيمياوي المحسوب للعليقة.

\begin{tabular}{|c|c|c|}
\hline \% النمو عليقة & البادئ & المادة العلفية \\
\hline 65 & 60 & ذرة صفراء \\
\hline 30 & 35 & كسبة فول الصويا \\
\hline 4 & 4 & * * بريمكس \\
\hline 1 & 1 & زيت عباد الشمس \\
\hline 100 & 100 & *التحليل الكيميائي المحسوب \\
\hline 20.07 & 22.08 & البروتين الخام (\%) \\
\hline 2999.5 & 2954 & الطاقة/الممثلة(كيلوسعرة/ كفم \\
\hline 0.97 & 1.22 & اللايسين (\%) \\
\hline 0.59 & 0.51 & المثيونين (\%) \\
\hline 0.39 & 0.77 & المثيونين + السستين (\%) \\
\hline 0.73 & 0.75 & الكالسيوم (\%) \\
\hline 0.54 & 0.55 & الفسفور المتيسّر (\%) \\
\hline
\end{tabular}

"التحليل الكيمياوي حسب (12) *"المركز البروتيني (بريمكس) من انتاج شركة الولاء

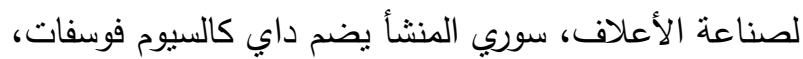

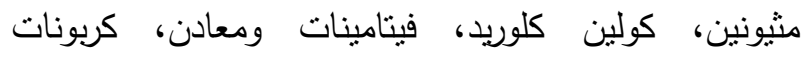
الكالسيوم، مضاد اكسدة، مضاد فطري، مضاد كوكسيديا، ملح طعام. حلات النتائج احصائياً بأستخدام نظام SAS (14) بأستخدام التصميم العشوائي CRD وقورنت الفروق المعنوية بين المتوسطات حسب Duncan (8). 
. $11,7 ، 6)$

جاول 2 ـ تأثير الوزن المتوسط والثقيل لفروج اللحم بعمر يوم واحد في معدل وزن الجسم الاسبوعي (غم) لسلالتي *Arbor Acres وتضريبهما (المتوسطد الخطأ القياسي)

\begin{tabular}{|c|c|c|c|c|c|c|}
\hline \multicolumn{6}{|c|}{ المتوسطدالخطأ القياسي } & \multirow[b]{2}{*}{ المعاملات } \\
\hline الاسبوع 6 & الاسبوع 5 & الاسبوع 4 & الاسبوع 3 & الاسبوع 2 & الاسبوع 1 & \\
\hline $2695.42 \pm 56.79^{\mathrm{ab}}$ & $2107.09 \pm 7.46^{b}$ & $1461.66 \pm 4.44^{\mathrm{c}}$ & $887.91 \pm 3.98^{c}$ & $431.75 \pm 12.12^{b c}$ & $155.71 \pm 1.93^{b}$ & T1 \\
\hline $2548.41 \pm 59.94^{b}$ & $2068.33 \pm 7.57^{c}$ & $1418.88 \pm 7.14^{d}$ & $930.55 \pm 3.59^{\mathrm{a}}$ & $452.50 \pm 2.08^{\mathrm{ab}}$ & $170.86 \pm 2.88^{\mathrm{a}}$ & $\mathbf{T} 2$ \\
\hline $2801.39 \pm 49.74^{\mathrm{a}}$ & $2189.73 \pm 14.44^{\mathrm{a}}$ & $1535.83 \pm 7.12^{\mathrm{a}}$ & $914.11 \pm 4.47^{b}$ & $405.27 \pm 5.97^{d}$ & $140.55 \pm 1.80^{\mathrm{c}}$ & T3 \\
\hline $2562.81 \pm 17.32^{b}$ & $2064.28 \pm 6.74^{c}$ & $1465.33 \pm 7.63^{c}$ & $908.73 \pm 3.18^{b}$ & $433.33 \pm 2.19^{b c}$ & $153.57 \pm 0.85^{b}$ & T4 \\
\hline $2750.74 \pm 54.40^{a}$ & $2193.33 \pm 6.80^{a}$ & $1504.94 \pm 2.81^{b}$ & $938.12 \pm 4.25^{\mathrm{a}}$ & $471.87 \pm 5.18^{\mathrm{a}}$ & $169.37 \pm 3.37^{a}$ & T5 \\
\hline $2799.04 \pm 40.79^{a}$ & $2106.05 \pm 5.33^{b}$ & $1434.37 \pm 4.44^{d}$ & $875.62 \pm 4.63^{c}$ & $425.00 \pm 8.38^{\mathrm{cd}}$ & $156.66 \pm 1.24^{b}$ & T6 \\
\hline * & $*$ & * & * & * & * & المعنوية \\
\hline
\end{tabular}

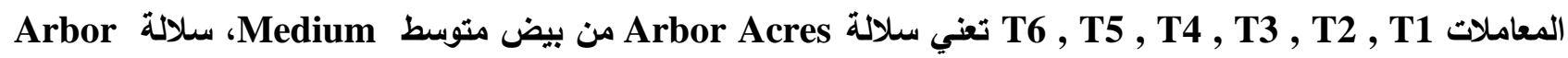

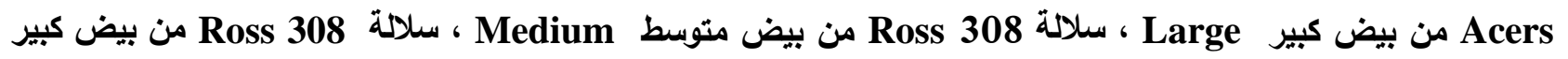

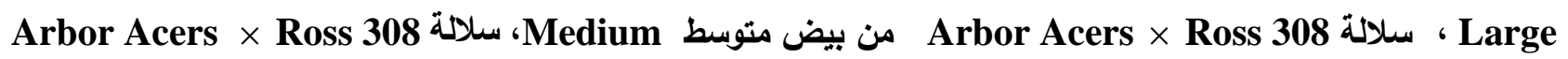
من بيض كبير الاربل * الاحرف المختلفة ضمن العمود الواحد تشثير الى وجود فروق معنوية بين متوسطات المعاملات على مستوى (P>0.05)

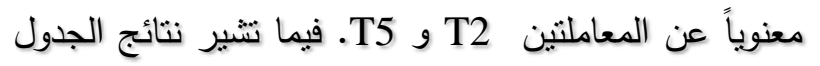
وتثير نتائج الجدول (3) الى معدل الزيادة الوزنية الاسبوعية (5) عدم وجود فارق معنوي بين معاملات التجربة للاسابيع اذ تفوقت المعاملة T2 و T5 على معاملات التجربة الاخرى 1،5،6 في معامل التحويل الغذابي الا انه يلاحظ تفوق معنوي(P<0.05) للمعاملتين T1 T2 T3 T2 Tلى المعاملة T2 حيث سجلت احسن معامل تحويل غذائي وكانت 1.55 و 1.60 كغم علف / كغم وزن حي على النوالي ولم تختلف Molenaar معنياً عن باقي معاملات التجربة وهذا مابينه و اخرون (11) من عدم وجود فروق معنوية لوزن الفرخ في كفاءة التحويل الغذائي. وتتير نتائج الجدول (6) الى تفوقاً

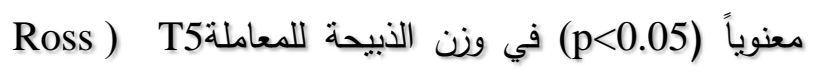
308 تضريب Arbor Acres منوسط الوزن) على المعاملة متوسط الوزن) ولم تختلف عن باقي Arbor Acres)T1 المعاملات وسجلت اعلى وزن ذبيحة 1875 غرام ولم تلاحظ فروق معنوية بين معاملات التجربة بالنسبة لقطعتي الصدر والافخاذ فيما سجلت المعاملة T2 اعلى نسبة تصافي للاناث بين المعاملات وكانت 78.1\% تلنها المعاملة T6 وسجلت 75 ولم تختلف معنوياً عن المعاملات T3 و T5 Tيما T2 اعطت المعاملتين T1 و T4 اقل نسبة نسبة تصافي وكانت 67.3 و 65.4\% .ومن نتائج الجدول (7) نلاحظ عدم وجود فروق معنوية في وزن الذبيحة والقطعيات الرئيسية خلال الاسبوع الاول فيما سجلت المعاملة T5 اعلى زيادة وزنية خلال الاسبوع الثاني وكانت 302.50 غرام فيما اعطت المعاملة T3 اعلى معدل زيادة وزنية خلال الاسبوعين الثالث والرابع وسجلت 508.83 و 621.72 غرام على التوالي فيما عادت المعاملة T5 الى التقوق في الاسبوع الخامس 688.89 غرام. اما خلال الاسبوع السادس فقد تفوقت المعاملة T6 على المعاملتين T2 وT4 ولم تختلف معنوياً عن المعاملات T1 و T3 وT5 ـ اما الزيادة الوزنية الكلية فقد تفوقت المعاملات T3 و T5 و T6 معنوياً و 2759.19 و 2749.52 (P<0.05) 2708.71 غرام على التوالي بالمقارنة مع المعاملتين T2 و T4 وقد يعزى التفوق للمعاملات T3 و T5 و T6 الى T6 اختلاف القابيلة الوراثية لكل سلالة وتداخل ذلك مع مؤامتها للبيئة وانعكست على تحسين كفاءة تحويل العلف ومن ثم زيادة معدل الوزن الحي وهذا مالاحظه وآخرون (2) حيث تفوقت سلالة لوهمان على السلالثنين فاوبرو ويوربرد. اما جدول (4) فقد تفوقت معنوياً (P<0.05) المعاملة T6 في معدل استهالك العلف الكلي ولم تختلف 
وكذللك لم تختلف المعاملات في وزن الكبد والقلب، اما بالنسبة للذكور ومن نفس الجدول (8) نلاحظ تفوق للمعاملة T2 في وزن القانصة والمعاملة T3 في وزن الكبد والمعاملة T1 في وزن القلب. نستتنج من هذه الدراسة تحسن الاداء الانتاجي لفروج اللحم عند تربية الافراخ ذات الاوزان المتوسطة للهجن التجارية وكذلك عند التضريب بين الهجن التجارية.
والثانوية الا انه لوحظ تقوق معنوي (0.05) المعاملتين و T5 في نسبة التصافي للذكور وسجلتا 74.2\% و 74\% على التوالي على باقي المعاملات وهذا مالاحظه وآخرون (11) من انه ليس هناك تاثير لوزن الفرخ على النسبة المئوية لقطعيات الذبيحة ونسبة التصافي. ومن الجدول (8) نلاحظ تفوقاً معنوياً (0.05) في وزن القانصة للاناث للمعاملة T2 معنوياً على المعاملتين T2 و T5 ولم تختلف معنوياً عن المعاملات T1 و T3 Tو Tو Th

جدول 3 ـ تأثيرالوزن المتوسط و الثقيل لفروج اللحم بعر يوم واحد في معدل الزيادة الوزنية (غم) لسلالتي Ross و Arbor *Acers

\begin{tabular}{|c|c|c|c|c|c|c|c|}
\hline \multicolumn{7}{|c|}{ المتوسط × الخطأ القياسي } & \multirow[b]{2}{*}{ المعاملات } \\
\hline (6-1) اسبوع) & الاسبوع 6 & الاسبوع 5 & الاسبوع 4 & الاسبوع 3 & الاسبوع 2 & الاسبوع 1 & \\
\hline $2655.44 \pm 57.05 \mathrm{a}^{\mathrm{b}}$ & $588.33 \pm 50.09^{\mathrm{ab}}$ & $645.42 \pm 3.35^{b}$ & $573.75 \pm 5.53^{b}$ & $456.15 \pm 12.17^{\text {bc }}$ & $276.04 \pm 10.21^{b}$ & $115.73 \pm 2.25^{b}$ & T1 \\
\hline $2501.76 \pm 61.13^{b}$ & $480.08 \pm 62.76^{b}$ & $649.45 \pm 5.78^{c}$ & $488.33 \pm 10.24^{\mathrm{c}}$ & $478.05 \pm 2.08^{b}$ & $281.63 \pm 3.32^{b}$ & $124.21 \pm 1.67^{\mathrm{a}}$ & $\mathbf{T 2}$ \\
\hline $2759.19 \pm 51.22^{\mathrm{a}}$ & $611.66 \pm 41.62^{a b}$ & $653.89 \pm 7.95^{\mathrm{c}}$ & $621.72 \pm 11.47^{a}$ & $508.37 \pm 5.82^{\mathrm{a}}$ & $264.72 \pm 5.37^{b}$ & $98.35 \pm 3.36^{d}$ & T3 \\
\hline $2541.45 \pm 17.02^{b}$ & $498.53 \pm 11.57^{b}$ & $598.95 \pm 2.16^{\mathrm{d}}$ & $556.60 \pm 10.77^{b}$ & $475.39 \pm 2.72^{b}$ & $279.76 \pm 1.36^{b}$ & $105.21 \pm 1.61^{\mathrm{cd}}$ & T4 \\
\hline $2708.71 \pm 53.74^{\mathrm{a}}$ & $557.41 \pm 60.95^{a b}$ & $688.89 \pm 7.20^{\mathrm{a}}$ & $566.31 \pm 6.74^{b}$ & $466.25 \pm 8.62^{b c}$ & $302.50 \pm 5.07^{a}$ & $127.35 \pm 3.16^{\mathrm{a}}$ & T5 \\
\hline $2749.52 \pm 41.20^{\mathrm{a}}$ & $692.99 \pm 43.17^{\mathrm{a}}$ & $671.67 \pm 3.94^{b}$ & $558.75 \pm 8.70^{b}$ & $450.62 \pm 4.01^{c}$ & $268.33 \pm 7.17^{b}$ & $107.14 \pm 0.26^{\mathrm{c}}$ & T6 \\
\hline * & * & $*$ & * & * & $*$ & $*$ & معنتوية \\
\hline
\end{tabular}

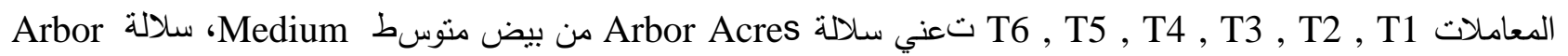

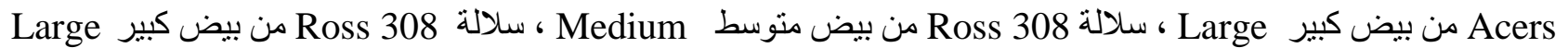
، سلالة Arbor Acers × Ross 308 من بيض منوسط Medium، سلالة Arbor Acers × Ross 308 من بيض Large كبير

** الاحرف المختلفة ضمن العمود الواحد نتير الى وجود فروق معنوية بين متوسطات المعاملات على مستوى (P>0.05) جدول 4. تأثير الوزن المتوسط والثقيل لفروج اللحم بعمر يوم واحد في معدل استهلاك العلف (غم)لسلالتي * Acres

\begin{tabular}{|c|c|c|c|c|c|c|c|}
\hline \multicolumn{7}{|c|}{ (المتوسطد الخطأ القياسي) } & \multirow[b]{2}{*}{ المعاملات } \\
\hline الكلية - الكية & الاسبوع 6 & الاسبوع 5 & الاسبوع 4 & الاسبوع 3 & الاسبوع 2 & الاسبوع 1 & \\
\hline $4260.10 \pm 77.59^{\mathrm{bc}}$ & $1186.50 \pm 56.08^{b}$ & $1016.4 \pm 16.60$ & $851.66 \pm 11.26^{b}$ & $644.16 \pm 11.30^{b}$ & $394.16 \pm 7.21^{b}$ & $167.14 \pm 3.45^{b}$ & T1 \\
\hline $4460.30 \pm 97.60^{\mathrm{ab}}$ & $1191.40 \pm 56.96^{b}$ & $1061.11 \pm 76.42$ & $800.25 \pm 14.28^{c}$ & $772.66 \pm 10.20^{\mathrm{a}}$ & $441.00 \pm 5.34^{\mathrm{a}}$ & $193.84 \pm 3.29 \mathrm{a}$ & T2 \\
\hline $4203.50 \pm 54.44^{\mathrm{c}}$ & $1190.00 \pm 63.36^{b}$ & $1020.00 \pm 10.12$ & $806.66 \pm 15.02^{c}$ & $640.20 \pm 11.43^{b}$ & $393.33 \pm 5.50^{b}$ & $153.33 \pm 3.85^{\mathrm{c}}$ & T4 \\
\hline $4486.90 \pm 91.30^{\mathrm{ab}}$ & $1200.00 \pm 76.86^{b}$ & $1100.22 \pm 12.82$ & $922.22 \pm 12.63^{a}$ & $631.87 \pm 10.69^{b}$ & $450.55 \pm 15.27^{a}$ & $182.00 \pm 4.45^{\mathrm{a}}$ & T5 \\
\hline $4584.20 \pm 60.86^{\mathrm{a}}$ & $1360.50 \pm 22.81^{\mathrm{a}}$ & $1107.01 \pm 62.96$ & $925.00 \pm 14.58^{\mathrm{a}}$ & $665.69 \pm 9.05^{b}$ & $390.50 \pm 7.69^{b}$ & $135.5 \pm 3.25^{\mathrm{c}}$ & T6 \\
\hline
\end{tabular}

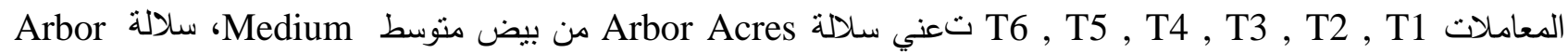

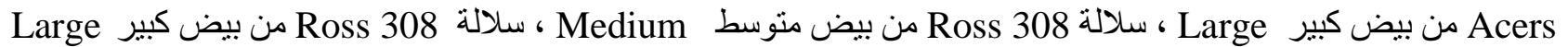

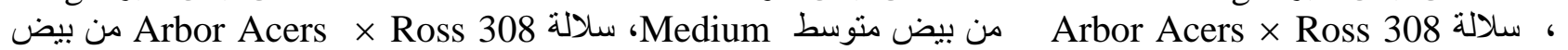
** الاحرف المختلفة ضمن العمود الواحد تشير الى وجود فروق معنوية بين متوسطات المعاملات على مسنوى (P>0.05) 
جدول 5. تأثير الوزن المتوسط والثقيل لفروج اللحم بعمر يوم واحد في معامل التحويل الغذائي (غم علف/غم زيادة وزنية) لسلالتي Ross و Arbor Acres وتضريبهما(المتوسطد الخطأ القياسي)

\begin{tabular}{|c|c|c|c|c|c|c|c|}
\hline \multicolumn{7}{|c|}{ (المتوسطد الخطأ القياسي) } & \multirow[b]{2}{*}{ المعاملات } \\
\hline الكلية & الاسبوع 6 & الاسبوع 5 & الاسبوع 4 & الاسبوع 3 & الاسبوع 2 & الاسبوع 1 & \\
\hline $1.60 \pm 0.04^{b}$ & $2.03 \pm 0.14$ & $1.57 \pm 0.03$ & $1.48 \pm 0.03^{b}$ & $1.41 \pm 0.01^{b}$ & $1.43 \pm 0.03^{\mathrm{abc}}$ & $1.44 \pm 0.06$ & T1 \\
\hline $1.78 \pm 0.01^{\mathrm{a}}$ & $2.53 \pm 0.22$ & $1.63 \pm 0.13$ & $1.64 \pm 0.04^{a}$ & $1.61 \pm 0.02^{\mathrm{a}}$ & $1.56 \pm 0.03^{\mathrm{a}}$ & $1.56 \pm 0.00$ & T2 \\
\hline $1.55 \pm 0.03^{b}$ & $2.06 \pm 0.18$ & $1.55 \pm 0.04$ & $3.8 \pm 0.01^{b}$ & $1.28 \pm 0.01^{c}$ & $1.33 \pm 0.05^{c}$ & $1.50 \pm 0.07$ & T3 \\
\hline $1.67 \pm 0.02^{\mathrm{ab}}$ & $2.38 \pm 0.12$ & $1.70 \pm 0.02$ & $1.45 \pm 0.04^{b}$ & $1.34 \pm 0.02^{b c}$ & $1.40 \pm 0.02^{\mathrm{bc}}$ & $1.4 \pm 0.01$ & T4 \\
\hline $1.65 \pm 0.06^{\mathrm{ab}}$ & $2.23 \pm 0.37$ & $1.59 \pm 0.00$ & $1.62 \pm 0.03^{a}$ & $1.35 \pm 0.04^{\mathrm{bc}}$ & $1.49 \pm 0.06^{\mathrm{ab}}$ & $1.42 \pm 0.01$ & T5 \\
\hline $1.66 \pm 0.02^{\mathrm{ab}}$ & $2.02 \pm 0.12$ & $1.64 \pm 0.09$ & $1.65 \pm 0.00^{\mathrm{a}}$ & $1.37 \pm 0.00^{b}$ & $1.45 \pm 0.00^{\mathrm{abc}}$ & $1.43 \pm 0.00$ & T6 \\
\hline$*$ & N.S & N.S & * & $*$ & * & N.S & المعنوية \\
\hline
\end{tabular}

Arbor تعني سلالة Arbor Acres T6 , T5 , T4 , T3 , T2 , T1 من بيض منوسط Medium، سلالة

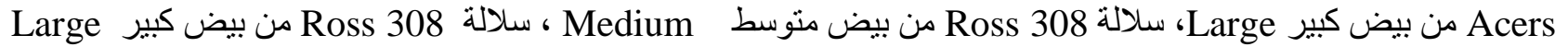

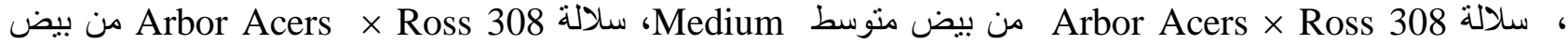
كبير Large ** الاحرف المختلفة ضمن العمود الواحد تثير الى وجود فروق معنوية بين منوسطات المعاملات على مستوى (P<0.05) جدول 6 ـ تأثير الوزن المتوسط والثقيل لفروج اللحم بعمر يوم واحد في الوزن النسبي للقطعيات الرئيسية والثانوية ونسبة التصافي للاناث لسلالتي Arbor AcresوRoss وتضريبهما (المتوسطد الخطأ القياسي)

\begin{tabular}{|c|c|c|c|c|c|c|c|}
\hline \multicolumn{7}{|c|}{ الصفات المدروسة } & \multirow[b]{2}{*}{ المعاملات } \\
\hline نسبة التصافي & الرقبة \% & الاجنحة \% & الظهر \% & الافخاذ \% & الصدر \% & وزن الأبيحة & \\
\hline $67.3 \pm 0.98^{c}$ & $6.2 \pm 0.56^{\mathrm{ab}}$ & $10.5 \pm 0.30$ & $20.1 \pm 1.19^{a b}$ & $28.7 \pm 2.30$ & $34.3 \pm 1.52$ & $1615 \pm 93.40^{b}$ & T1 \\
\hline $78.1 \pm 0.47^{\mathrm{a}}$ & $5.8 \pm 0.10^{\mathrm{ab}}$ & $9.8 \pm 0.01$ & $20.5 \pm 0.40^{\mathrm{a}}$ & $26.4 \pm 0.50$ & $36.5 \pm 2.07$ & $1720 \pm 57.73^{a b}$ & $\mathrm{~T} 2$ \\
\hline $73.0 \pm 0.56^{b}$ & $5.8 \pm 0.03^{\text {ab }}$ & $10.6 \pm 0.57$ & $19.5 \pm 0.34^{\text {abc }}$ & $29.0 \pm 1.12$ & $34.9 \pm 1.51$ & $1790 \pm 72.11^{\mathrm{ab}}$ & T3 \\
\hline $65.4 \pm 0.93^{c}$ & $5.0 \pm 0.50^{b}$ & $10.8 \pm 0.00$ & $18.2 \pm 0.52^{\mathrm{bcd}}$ & $26.1 \pm 0.57$ & $39.7 \pm 1.46$ & $1733 \pm 92.79^{a b}$ & T4 \\
\hline $72.10 \pm 1.60^{b}$ & $6.9 \pm 0.56^{a}$ & $10.1 \pm 0.54$ & $17.8 \pm 0.55^{\mathrm{cd}}$ & $27.7 \pm 1.20$ & $37.30 \pm 2.03$ & $1875 \pm 54.87^{a}$ & T5 \\
\hline $75.0 \pm 1.32^{b}$ & $6.0 \pm 0.59^{a b}$ & $10.9 \pm-.54$ & $16.9 \pm 0.02^{\mathrm{a}}$ & $26.0 \pm 1.73$ & $40.00 \pm 1.15$ & $1650 \pm 57.73^{\mathrm{ab}}$ & T6 \\
\hline * & $*$ & N.S & $*$ & N.S & N.S & * & المنتوية \\
\hline
\end{tabular}

Arbor تعني سلالة Arbor Acres , T5 , T4 , T3 , T2 , T1 من بيض منوسط Medium، سلالة ،

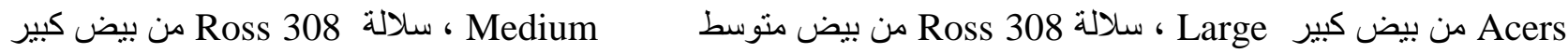

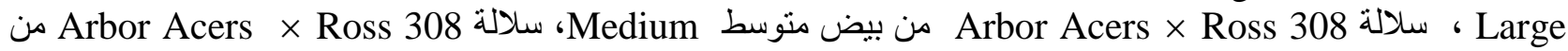

Lيض كبير Large

* * الاحرف المختلفة ضمن العمود الواحد نتير الى وجود فروق معنوية بين متوسطات المعاملات على مستوى (P<0.05) جدول 7 ـ تأثير الوزن المتوسط والثقيل لفروج اللحم بعمر يوم واحد في الوزن النسبي للقطعيات الرئيسية والثانوية ونسبة التصافي للاكور لسلالتي Arbor AcresوRoss وتضريبهما (المتوسطد الخطأ القياسي)

\begin{tabular}{|c|c|c|c|c|c|c|c|}
\hline \multicolumn{7}{|c|}{ الصفات المدروسة } & \multirow[b]{2}{*}{ المعاملات } \\
\hline نسبة التصافي & الرقبة \% & الاجنحة \% & الظهر \% & الافخاذ \% & الصدر \% & وزن الأبيحة & \\
\hline $74.0 \pm 0.55^{\mathrm{a}}$ & $5.8 \pm 0.57$ & $9.4 \pm 0.17$ & $18.4 \pm 0.31$ & $25.8 \pm 1.15$ & $40.50 \pm 1.15$ & $2000 \pm 125.83$ & T1 \\
\hline $70.0 \pm 0.86^{b}$ & $5.9 \pm 0.62$ & $10.7 \pm 0.57$ & $18.8 \pm 0.49$ & $27.1 \pm 1.73$ & $37.4 \pm 2.15$ & $2100 \pm 173.20$ & T2 \\
\hline $71.3 \pm 0.57^{b}$ & $5.2 \pm 0.41$ & $10.2 \pm 0.57$ & $17.0 \pm 0.52$ & $28.0 \pm 1.00$ & $39.6 \pm 1.74$ & $2210 \pm 66.58$ & T3 \\
\hline $70.0 \pm 0.86^{b}$ & $6.4 \pm 0.24$ & $9.5 \pm 0.30$ & $18.2 \pm 0.52$ & $27.4 \pm 1.13$ & $38.5 \pm 2.28$ & $2210 \pm 49.32$ & T4 \\
\hline $74.2 \pm 0.57^{\mathrm{a}}$ & $5.8 \pm 0.03$ & $10.9 \pm 0.53$ & $18.5 \pm 0.88$ & $28.9 \pm 2.30$ & $35.8 \pm 2.23$ & $2300 \pm 202.07$ & T5 \\
\hline $\begin{array}{c}71.0 \pm 0.57^{b} \\
*\end{array}$ & $\begin{array}{l}5.0 \pm 0.28 \\
\text { N.S }\end{array}$ & $\begin{array}{l}10.8 \pm 0.46 \\
\text { N.S }\end{array}$ & $\begin{array}{c}17.0 \pm 0.28 \\
\text { N.S }\end{array}$ & $\begin{array}{c}30,2 \pm 1.23 \\
\text { N.S }\end{array}$ & $\begin{array}{c}37.0 \pm 0.57 \\
\text { N.S }\end{array}$ & $\begin{array}{c}2000 \pm 115.47 \\
\text { N.S }\end{array}$ & مستوى المعنوية \\
\hline
\end{tabular}

Arbor Acers تعني سلالة Arbor Acres T6 , T5 , T4 , T3 , T2 , T1 من بيض منوسط Medium، سلالة

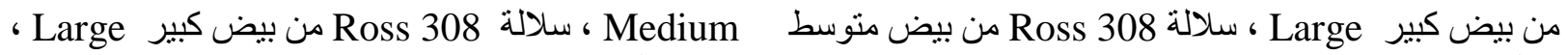
سلالة Arbor Acers × Ross 308 من بيض منوسط Medium، سلالة Arbor Acers × Ross 308 من بيض 
جدول 8. تأثير الوزن المتوسط والثقيل لفروج اللحم بعر يوم واحد في الوزن النسبي للاحشاء الداخلية المأكولة للاناث *Arbor AcresوRoss وتضريبهما(المتوسطد الخطأ القياسي)

\begin{tabular}{|c|c|c|c|c|c|c|}
\hline \multicolumn{3}{|c|}{ الأكور } & \multicolumn{3}{|c|}{ الاناث } & \multirow[b]{2}{*}{ المعاملات } \\
\hline القلب \% & الكبا \% & القانصة \% & القلب \% & الكبا \% & القانصة \% & \\
\hline $0.57 \pm 0.01^{\mathrm{a}}$ & $2.79 \pm 0.05^{\mathrm{ab}}$ & $1.30 \pm 0.17^{b}$ & $0.57 \pm 0.01$ & $2.50 \pm 0.28$ & $1.70 \pm 0.15^{\mathrm{ab}}$ & T1 \\
\hline $0.46 \pm 0.01^{\mathrm{ab}}$ & $2.44 \pm 0.22^{b}$ & $1.90 \pm 0.23^{a}$ & $0.48 \pm 0.01$ & $2.44 \pm 0.25$ & $2.00 \pm 0.23^{a}$ & $\mathbf{T} 2$ \\
\hline $0.34 \pm 0.01^{b}$ & $2.99 \pm 0.23^{\mathrm{a}}$ & $1.26 \pm 0.03^{b}$ & $0.42 \pm 0.05$ & $2.45 \pm 0.25$ & $1.93 \pm 0.22^{\mathrm{ab}}$ & T3 \\
\hline $0.44 \pm 0.01^{\mathrm{ab}}$ & $1.87 \pm 0.07^{c}$ & $1.46 \pm 0.11^{b}$ & $0.51 \pm 0.02$ & $2.76 \pm 0.05$ & $1.44 \pm 0.12^{b}$ & T4 \\
\hline $0.52 \pm 0.01^{\mathrm{ab}}$ & $2.72 \pm 0.13^{\mathrm{ab}}$ & $1.39 \pm 0.05^{b}$ & $0.50 \pm 0.02$ & $3.13 \pm 0.13$ & $1.45 \pm 0.02^{b}$ & T5 - \\
\hline $0.44 \pm 0.12^{\mathrm{ab}}$ & $2.37 \pm 0.11^{b}$ & $1.57 \pm 0.06^{\mathrm{ab}}$ & $11 j 0 ر 58 j 0$ & $2.17 \pm 0.14$ & $1.74 \pm 0.05^{\mathrm{ab}}$ & T6 \\
\hline * & * & * & N.S & N.S & * & مستوبية \\
\hline
\end{tabular}

المعاملات Arbor Acres تعني سلاتلة T6 , T5 , T4 , T3 , T2 , T1 من بيض منتوسط Medium، سلالة Arbor Acers ، سلة

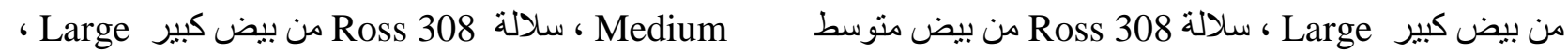

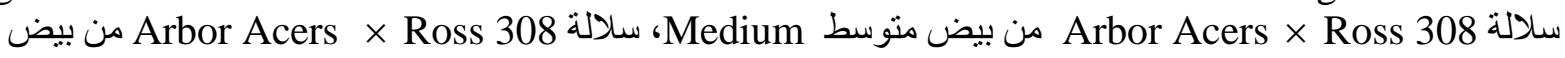
كبير Large ** الاحرف المختلفة ضمن العمود الو احد تثير الى وجود فروق معنوية بين منوسطات المعاملات على مسنوى (P<0.05)

\section{REFERENCES:}

1.Ahmed , L.S. 2008 . Evaluated the Productive Performance of two Broiler Parent Strains and Their Progeny . M.Sc. Thesis, College of Agriculture .University of Salah AL-Deen.

2. AL -Khailany, F.M,A.A.K.AL-Athary and H.J.AL-Daraji .2003 .The effect of Force resting on product performance of progeny from different strains of broiler parents.Iraqi Agriculture Journal (special Issu ), 8 (1).

3. Alzawbaii, R.J.R.2010. Effect of Hatching Egg Weight on Fertility ,Hatchability and Subsequent Broiler Performance .M.Sc. college of Agriculture .University of Baghdad. 4.Abiola, S.S., O. Meshioye, O. Oyerind and M. A., Bamgbose, 2008. Effect of egg size on hatchability of broiler chick. Archives De Zootecnia .Vol.57, N. 217.P.85.

5.Constantini ,F., and F. Panella, 1984. Correlations between egg weight ,chick weight and broilers performance. Animal breed. 51:35-40.

6.Deeming, D.C., 2000. What is chick quality? Worlds Poult. Sci.,34-35.

7.Deeming, D.C.,2005.Yolk sac body dimensions and hatching quality of chicks, ducklings and poults. Br. Poult. Sci.,46:560564.

8.Duncan, D.D.,1955. Multiple range and multiple F-test. Biometrices., 11:1-42.

9.Falconer, D. S., 1981. Introduction to Quantitative Genetics.2 ${ }^{\text {nd }}$ Edition. Logman .New York.

10.Laughlin, K. F. 2005. Management band control of egg size; International Hatchery Practice.

Volume 19 Number 8.

11.Molenaar, R. M., M. Reijrink, R. Meijerhof and Van H. den Brand. 2008. Relationship between hatching length and weight on later production performance in broilers. Journal Poult. Sci., Vol,64:599-604.

12.National Research Council (NRC)1994. Nutrient Requirements of Poultry. 9th rev. ed. National Academy Press, Washington. DC.

13.Oblakova ,M.M., Lalev.P., Hristakieva and Georgieva, 2008. Effect of weight on the production traits of hatched turkey poults not later than 16 weeks of age. Tuakia Journal of Science, Vol.6,No.4, P:83-87.

14.SAS 2009.Users Guide: Statistics, Release Edition.SAS institute Inc. Cary ,NC. Ver q.1.

15. Tesseraud, S., R.A.E. Pym,E. Lebihan -Duval and M.J. Duclos, 2003. Response 
of broilers selected on carcass quality to dietary supply live performance, muscle development and circulating insulin - like growth factor. Poultry Sci., 82:1011-1016.

16.Willemsen, H., N. Everraet, A. Witter, S.Desmit, M. Debonnc, F.Verschuerc, P.Garain, D. Berckmans and V. Bruggeman
,2008. Cirtical assessment of chick quality measurements as an indicator of post hatch performance. Poultry Sci., 87:2358-2366.

17.Wolanski,N. J., R. A. Renema.,F.E. Robinson, V.L. Carney and B. L. Fanche r,2007.Relationship among egg characteristics chick measurement and early growth traits in ten broiler breeder strains. Poult. Sci.,86:17841792. 